\title{
La actividad filosófica según Wittgenstein. \\ Confusiones conceptuales e investigación gramatical ${ }^{2}$
}

\author{
Wittgenstein's philosophical activity. \\ Conceptual confessions and grammatical research
}

\section{Resumen}

El presente artículo pretende ahondar en la distinción que Wittgenstein señala en las Investigaciones Filosóficas sobre la explicación y la descripción como formas diferenciadas de abordar disímiles objetos de estudio. Diferencia a partir de la cual, a su vez, es posible dilucidar la concepción y el método que el autor plantea para la acción propiamente filosófica como investigación gramatical, por cuanto su labor se ejercería sobre conceptos y confusiones conceptuales en contraposición a las tendencias generalizantes que el cientificismo pretende universalizar.

Palabras clave: : labor filosófica, explicar, describir, investigación gramatical, ansia de generalidad, juegos de lenguaje.

\section{Abstract}

This article aims to examine in detail the distinction made by Wittgenstein between the explanation and description of different ways to approach dissimilar subjects of study. This distinction was mae in the Philosophical Investigations. From this difference, it is possible to explain the concept and the method that the author suggests for the action purely philosophically. For example, grammatical research exercised on concepts and conceptual confusions in comparison with the generalizing trends that scientism aims to universalize.

Keywords: philosophicalwork, to explain, to describe, grammatical research, anxiety of generalization, language games.

\section{Recibido el 8 de junio de 2011 y aprobado el 12 de junio de 2011}

1 Oscar Orlando Espinel Bernal es docente e investigador de la Corporación Universitaria Minuto de Dios-Uniminuto y de la Universidad Pedagógica Nacional. Candidato a Magister en Educación de la Universidad Pedagógica Nacional, Especialista en Pedagogía del mismo centro universitario y miembro del Grupo de Investigación Pedagogía y Política de la Universidad Pedagógica Nacional. Correo electrónico: bespinelo@yahoo.es.

2 Texto de la ponencia presentada en el Coloquio de Estudiantes del Departamento de Filosofía de la Universidad Minuto de Dios realizado los días 16, 17 y 18 de Mayo de 2011. 


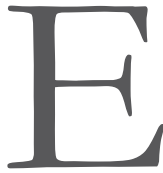

1 presente escrito se basa, primordialmente, en la propuesta filosófica esbozada en las Investigaciones Filosóficas de Ludwig Wittgenstein para revisar la diferencia que el autor presenta entre la descripción y la explicación dentro del tratamiento de las cuestiones filosóficas. Esta diferenciación permitirá analizar desde allí la concepción wittgensteiniana acerca de la filosofía y el método propuesto para ella.

Para este fin intentaré esclarecer lo que Wittgenstein entiende por una auténtica labor filosófica, contrastando, en cierto sentido, lo que este autor concibe como la forma tradicional de hacer filosofía y lo que él considera que es una práctica filosófica adecuada. De esta manera, pretendo explicitar el método propuesto por Wittgenstein, haciendo un recorrido desde la concepción de la filosofía como crítica del lenguaje presentada en el Tractatus Logico-Philosophicus, como paradigma de la vieja forma de abordar los problemas filosóficos, hasta llegar a la concepción de ésta como investigación gramatical en su periodo tardío y de manera particular en las Investigaciones Filosóficas.

En este punto hago especial énfasis en las razones que tiene Wittgenstein para afirmar la noción en la que los problemas filosóficos son problemas conceptuales y no empíricos, lo cual nos lleva a la idea de que para abordarlos es necesario recurrir a esta investigación gramatical.

\section{La labor filosófica desde la perspectiva wittgensteineana}

El trabajo filosófico de Wittgenstein y su concepción de la filosofía han dado nuevos virajes al quehacer filosófico. La filosofía wittgensteiniana, en su intento por alejarse de la noción y prácticas tradicionales de la filosofía, se convierte en un tipo de pensamiento bastante innovador y fecundo con ideas revolucionarias acerca de la manera de ver y tratar los problemas filosóficos. Un nuevo concepto de la filosofía y un nuevo método para ella es la propuesta del Wittgenstein tardío: la filosofía como actividad terapéutica y su método, la investigación gramatical.

Wittgenstein concibe a la filosofía como una actividad y no como una doctrina o teoría. La filosofía debe encargarse de buscar una acertada comprensión de los problemas mediante la aclaración de los conceptos que se emplean en el planteamiento del problema. El filósofo de Viena se aleja así de la tradicional práctica de elaborar teorías basadas en generalizaciones indebidas, a través de las cuales se pretendía explicar de manera definitiva alguna cuestión en particular.

Siguiendo este método, Wittgenstein pretende mostrar que los problemas filosóficos no son auténticos problemas, sino pseudo-problemas que se deben a confusiones en el lenguaje, producidos por generalizaciones inapropiadas, incorrectas analogías y extensiones indebidas de los 
conceptos a regiones que no son las propias. Por esta razón, el filósofo no debe intentar solucionar estos problemas ya que no requieren de solución sino de una adecuada clarificación.

\section{La teoría figurativa: el lenguaje como imagen del mundo}

Las Investigaciones Filosóficas contienen una profunda crítica, aunque no un total rechazo, a las principales tesis expuestas en el Tractatus. Dicha crítica se dirige principalmente a la reducción del lenguaje a una función puramente descriptiva. Esta situación es consecuencia inevitable de la aceptación de los supuestos seguidos a lo largo de esta obra perteneciente a la primera época dentro de la labor filosófica wittgensteiniana. Entre tales supuestos existe uno muy básico, a saber, la postulación del isomorfismo lógico entre el lenguaje y el mundo o teoría figurativa de la proposición, en donde el lenguaje es un espejo lógico de la realidad.

En el prólogo al Tractatus, Wittgenstein anuncia el objetivo principal de esta obra declarando en el prólogo:

El libro trata los problemas filosóficos y muestra -según creo- que el planteamiento de estos problemas descansa en la incomprensión de la lógica de nuestro lenguaje. Cabría acaso resumir el sentido entero del libro en las palabras: lo que siquiera puede ser dicho, puede ser dicho claramente; y de lo que no se puede hablar hay que callar. El libro quiere, pues, trazar un límite al pensar o, más bien, no al pensar, sino a la expresión de los pensamientos: porque para trazar un límite al pensar tendríamos que poder pensar ambos lados de este límite (tendríamos, en suma, que poder pensar lo que no resulta pensable). Así pues, el límite sólo podrá ser trazado en el lenguaje, y lo que reside más allá del límite será simplemente absurdo (Wittgenstein, 2003, p. 47).
De esta manera, el Wittgenstein del Tractatus intenta revisar los problemas que se plantea la filosofía, pues considera que la mayoría de ellos son malentendidos o confusiones, debido a una errónea comprensión de la lógica de nuestro lenguaje. Afirma Wittgenstein (2003): "La mayor parte de las proposiciones e interrogantes que se han escrito sobre cuestiones filosóficas no son falsas, sino absurdas. De ahí que no podamos dar respuesta en absoluto a interrogantes de este tipo, sino sólo constatar sus condición de absurdos" (p. 66).

En su opinión, lo que es posible ser pensado es de hecho expresable en el lenguaje; por tal razón, es necesario hacer un viraje hacia el lenguaje mismo, investigando su posibilidad y su lógica con el propósito de establecer todo aquello que puede ser dicho y trazar, así, los límites entre lo pensable y lo impensable ${ }^{3}$. Con ello se evitaría que en la filosofía se transgredieran dichos límites.

En el Tractatus, el lenguaje ha de ser visto, única y exclusivamente como representación de lo real. En la teoría tractariana del lenguaje como imagen, las proposiciones con sentido poseen una estructura lógica que refleja la propia estructura lógica de la realidad, ya que se supone que el lenguaje y el mundo poseen la misma arquitectura o estructura lógica. Podríamos explicar mejor esta idea mencionando que la tesis del isomorfismo lógico entre la realidad y el lenguaje declara que la posibilidad de que la proposición sea una figura de la realidad o de un estado de cosas se debe a que ha de haber algo común entre la figura y lo figurado. Aquello que comparten es precisamente la forma lógica, es decir, la manera como se relacionan los objetos en la realidad y los elementos simples del lenguaje ${ }^{4}$. Este isomorfismo lógico le permite llegar a afirmar que la proposición es una figura (Wittgenstein, 2003, p. 66) y como tal representa un posible

3 "Lo que en éste (el Tractatus) se desarrolla primordialmente es una teoría del pensamiento a través de una teoría del lenguaje [...] Se trata, pues, de establecer los límites entre lo pensable y lo impensable, entre el mundo y lo que queda fuera del mundo, mediante el establecimiento de los límites entre lo expresable y lo inefable o, lo que es lo mismo, entre el sentido y el sin sentido" (López, 1986, p. 18).

4 Ver Wittgenstein (2003), los aforismos 2.151 y 2.18. 
estado de cosas. De tal manera que mediante un conjunto o sistema de proposiciones podemos construir y expresar un modelo de la realidad.

El método empleado por Wittgenstein en el Tractatus para establecer el sentido o sin sentido de las proposiciones es el análisis o descomposición de las proposiciones hasta sus elementos simples 5 . En este punto hemos de decir que el autor del Tractatus sustenta su teoría del lenguaje en la existencia de objetos simples en la realidad que son representados por elementos simples o nombres en el lenguaje (Wittgenstein, 2003, p. 58). Para Wittgenstein (2003) es evidente que la realidad ha de estar compuesta por estos objetos simples, que combinados generan los estados de cosas que la conforman ${ }^{6}$.

Los objetos forman la sustancia del mundo. Por eso no pueden ser compuestos (p. 51).

Lo fijo, lo persistente y el objeto son uno y lo mismo (p. 52).

El objeto es lo fijo, persistente; la configuración es lo cambiante, inestable (p. 52).

Sin embargo, el nombre por sí solo aún no puede decir nada. Si los nombres expresan algo es porque se combinan entre sí formando una proposición. La combinación de estos nombres figuran una posible disposición de objetos, es decir, representan un estado de cosas de la realidad. El mundo no se nos presenta como un montón de objetos aislados, sino como un encadenamiento de hechos que, a su vez, surgen de la interrelación de estos objetos simples. "El mundo es la totalidad de los hechos, no de las cosas" (Wittgenstein, 2003, p. 49).
Por tal razón, una descripción del mundo es una descripción de los hechos que suceden en él y no un simple inventario de cosas u objetos tomados de manera aislada. Por consiguiente, las proposiciones que figuran los hechos son las que describen el mundo y no los nombres, ni un mero inventario de ellos.

Ya se ha dilucidado que la combinación de los nombres forman las proposiciones, pero hay que aclarar que dentro de éstas se encuentran, en términos generales, dos clases de proposiciones: proposiciones simples y proposiciones complejas. Las proposiciones complejas son funciones de verdad de las proposiciones que la componen. Si una proposición " $\mathrm{r}$ " es una función de verdad de "p", esto quiere decir que la verdad o falsedad de "r" está determinada por la verdad o falsedad de "p". En últimas, el carácter veritativo de una proposición depende de su relación con las proposiciones elementales y sería éste el sentido del análisis del lenguaje propuesto en el Tractatus: indagar las proposiciones para develar su estructura interna y así establecer su relación con las proposiciones elementales, las cuales le aportarían sus condiciones de verdad.

Recordemos que el objetivo primordial del análisis del lenguaje es presentarnos lo que puede ser dicho claramente. Según el Tractatus, todas las proposiciones auténticas pueden ser analizadas hasta llegar a proposiciones elementales ${ }^{7}$. Este análisis mostrará la naturaleza figurativa que se encuentra oculta en las proposiciones ordinarias.

Si dicho análisis es posible y los elementos simples a los que se llega nombran objetos del mundo real y están combinados de manera que representen algo posible, la proposición tendrá

5 Las proposiciones en el Tractatus son figuras de hechos que podrían ser analizadas hasta llegar a sus componentes últimos - los nombres correspondientes a objetos simples. "Si el mundo no tuviera sustancia alguna, el que una proposición tuviese sentido dependería de que otra proposición fuera verdadera" y entonces este proceso habría de seguirse ad infinitum. Ver los aforismos $2.02-2.0212$ de Wittgenstein (2003). Para más información acerca del análisis de las proposiciones en el Tractatus, ver Fann K.T. (1975).

6 "La substancia es lo que persiste independientemente de lo que es el caso" (Wittgenstein, 2003, p. 52). Wittgenstein sustentaba la posibilidad del mundo y de su representación en el lenguaje a partir de tales objetos simples y de su designación en el lenguaje a través de los nombres.

7 Ver Wittgenstein (2003), los aforismos 3.25, 3.251, 3.26. 
sentido, de lo contrario, la proposición carecerá de éste ${ }^{8}$. Tal método surge al interior de la teoría figurativa del lenguaje, según la cual éste se limita a representar, a describir el mundo. Una proposición con sentido pertenece a un lenguaje lógico o descriptivo. El lenguaje con sentido por excelencia es el empleado en las ciencias, el cual se limita a describir fenómenos empíricos, de tal manera que es posible determinar su verdad o falsedad según su concordancia con los hechos.

\section{El significado como uso frente al carácter figurativo del lenguaje}

Pero en las Investigaciones, inconforme con esta unilateral reducción, nuestro autor señala este carácter descriptivo como una entre muchas funciones del lenguaje, tales como: dar y recibir órdenes, relatar un acontecimiento o especular acerca de él, elaborar hipótesis, resolver un problema de aritmética, traducir de un lenguaje a otro, etc 9 .

Además de esto, existen en el lenguaje otros términos que a diario empleamos y que no tienen un referente en el mundo real; tal es el caso, de palabras como quizás, las cuales cumplen un papel bien definido dentro del lenguaje, y que, aunque no sean un nombre de un objeto real, no por ello dejan de ser significativas. Su sentido o significado está dado por su uso en el lenguaje y por su aplicación en situaciones particulares y de manera determinada. "El dominio del significado es mucho más amplio que el de la referencia" (López, 1986, p. 104). El lenguaje ya no es visto desde su carácter figurativo, sino primordialmente por su uso. El significado de una palabra es su uso en el lenguaje (Wittgenstein, 1988, p. 41).

Ahora bien, las palabras y las proposiciones no tienen establecido un uso único, sino que les damos una multiplicidad de usos de acuerdo al juego del lenguaje en que están imbricadas: "Llamaré también «juego de lenguaje» al todo formado por el lenguaje y las acciones con las que está entretejido" (Wittgenstein, 1988, p. 25); es decir, éstas adquieren significado dependiendo del contexto, sus relaciones con otras actividades y el propósito con el cual son usadas. En consecuencia, el significado no está instituido de manera a priori, sino que se obtiene a partir del uso de los conceptos en medio de un determinado contexto o juego de lenguaje inscrito en una forma particular de vida.

De esta manera es criticado el esencialismo perseguido $^{10}$ por el primer Wittgenstein en el Tractatus, en su pretensión de sustentar la tesis de la existencia de un único lenguaje lógico, del cual se derivaría el significado de cada palabra y el sentido o sin sentido de las proposiciones. Dicho de otra forma, para el primer Wittgenstein, la posibilidad del lenguaje de expresar algo se sostiene en la existencia de dicho lenguaje lógico. Es un esencialismo expresado en la búsqueda de una forma general de la proposición que le permite descubrir la esencia del lenguaje y las condiciones a priori de la verdad y del sentido de las proposiciones. No hay nada fijo en el lenguaje ni nada común en los diferentes juegos de lenguaje que les otorgue la calidad de lenguaje y que se pueda establecer como algo esencial.

8 Es importante recordar en este punto que la postulación de la existencia de nombres, que tienen la capacidad de remplazar o representar en el lenguaje los objetos del mundo real, es fundamental para hallar la relación entre el lenguaje y el mundo; formulado de otra manera: este postulado responde al interrogante de cómo el lenguaje puede hablar del mundo. Si hay una relación directa entre el nombre y los objetos, se podrá realizar un análisis de las proposiciones hasta llegar a estos elementos simples que representan de manera inmediata en el lenguaje a los objetos del mundo real. "En la proposición el nombre hace las veces del objeto" (Wittgenstein, 2003, p. 66).

9 En el §23 de las Investigaciones Filosóficas, Wittgenstein (1988) ofrece una lista de diferentes funciones que puede cumplir el lenguaje, lejos de ser puramente descriptivo como lo pretende el Tractatus. Y a continuación de mostrar esta pluralidad de usos del lenguaje hace el siguiente comentario: "Es interesante comparar la multiplicidad de herramientas del lenguaje y de sus modos de empleo, la multiplicidad de géneros de palabras y oraciones, con lo que los lógicos han dicho sobre la estructura del lenguaje. (Incluyendo el autor del Tractatus LogicoPhilosiphicus)" (p. 41).

10 Acerca de esta tesis sobre el esencialismo ver Van Deursen (1973). 
En vez de indicar algo que sea común a todo lo que llamamos lenguaje, digo que no hay nada en absoluto común a estos fenómenos por lo cual empleamos la misma palabra para todos -sino que están emparentados entre sí de muchas maneras diferentes. $\mathrm{Y}$ a causa de este parentesco, o de estos parentescos, lo llamamos a todos «lenguaje» (Wittgenstein, 1988, p. 87).

Este esencialismo se manifiesta también en la tesis del atomismo lógico, el cual, a su vez, se apoya en la idea de identidad entre la forma lógica del lenguaje y la de la realidad, es decir, en la teoría del isomorfismo lógico. Este atomismo, como ya se ha dicho, se basa en la existencia de objetos simples -figurados en el lenguaje por nombressobre los cuales se funda el hecho de que las proposiciones puedan expresar algo con sentido. Sin embargo, en las Investigaciones, el significado de la proposición no depende de la existencia de estos objetos simples, sino fundamentalmente, del uso que se le dé a las palabras y oraciones. El sentido de las proposiciones no se reduce a su naturaleza figurativa, sino al uso que de ellas se hace dentro de un contexto determinado. El significado no puede ni debe restringirse exclusivamente a esta estricta relación descriptiva entre el lenguaje y la realidad, promulgada por el Tractatus.

Wittgenstein (1988), en el parágrafo 11, compara las palabras con herramientas; al igual que éstas, las palabras se definen por su uso, el cual puede ser muy variado. Y en el parágrafo 15, enuncia que dar a las palabras una función meramente referencial es asumir que nombrar algo es similar a fijar un rótulo a una cosa (Wittgenstein, 1988, p. 29).

Las palabras no son meras etiquetas que se aplican a los objetos, sino instrumentos que cumplen funciones de muy distinto tipo. Su uniformidad es sólo aparente, el lenguaje es -dice Wittgenstein- como la cabina de una locomotora (Investigaciones Filosóficas, $\$ 12)$ en la que vemos una serie de mandos aparentemente iguales, cada uno de los cuales, no obstante, tiene un fin diferente (López, 1986, p. 110).

El lenguaje no es pues un instrumento que realice una única función o propósito esencial, sino que es una caja de herramientas que sirve para una extensa variedad de propósitos. Es posible que la herramienta tenga un uso o función habitual al cual los individuos de una comunidad están acostumbrados, pero nada impide que esa misma herramienta, en un momento determinado, asuma otros usos diferentes.

De esta manera se viene al piso la pretendida independencia de los elementos simples en el Tractatus. La palabra por sí sola no significa nada, ha de estar enlazada a un contexto, a un medio desde el cual se pronuncia y en el cual tiene una función singular. Esta aplicabilidad le otorga vida. Cada oración está inmersa dentro de un sistema de lenguaje.

Por otro lado, el lenguaje no está exento de variaciones ya que los significados están dados tanto por las condiciones lingüísticas como por las influencias extralingüísticas. El lenguaje es una actividad social y, por tanto, está sujeto a las condiciones de vida de quienes lo utilizan. "Las palabras son acciones y como tales se inscriben dentro de unos contextos, de unas situaciones y unas conductas que constituyen las formas de vida" (López, 1986, p. 117).

Y esa multiplicidad no es algo fijo de una vez por todas; sino que nuevos tipos de lenguaje, nuevos juegos de lenguaje, como podemos decir, nacen y otros envejecen y se olvidan... La expresión «juego de lenguaje» debe poner de relieve aquí que hablar el lenguaje forma parte de una actividad o de una forma de vida (Wittgenstein, 1988, p. 39).

Wittgenstein proclama en su etapa posterior a la del Tractatus que el verdadero método que permita disolver los problemas filosóficos debe dirigirse a los contextos particulares en los cuales las palabras son usadas para descubrir allí lo que él llama gramática. El verdadero método, dice Wittgenstein en el parágrafo 109, es aquel que ni explica ni deduce nada (Wittgenstein, 1988, p. 123), simplemente describe el fenómeno, poniéndolo frente a nuestros ojos. En el uso efectivo de los conceptos en casos particulares se develan las reglas con, o bajo las cuales, los conceptos son usados. 


\section{La investigación gramatical}

Hemos afirmado que para Wittgenstein los problemas filosóficos instalan sus raíces en diversas confusiones en el funcionamiento del lenguaje. Pues bien, el lenguaje puede llegar a confundirnos por medio de engañosas similitudes superficiales que tienen las palabras y las oraciones, las cuales conducen a realizar falsas analogías e inválidas generalizaciones. Es por ello que la investigación propone estudiar muy detenidamente el funcionamiento de nuestro lenguaje y de los conceptos, a fin de visualizar aquello que siempre hemos tenido ante nuestros ojos, es decir, la manera como realmente funcionan nuestros conceptos en el lenguaje.

Siguiendo este propósito, Wittgenstein realiza un cuidadoso estudio de casos concretos en los cuales son usados algunos conceptos que han sido motivo de largas discusiones y eternos malentendidos. Esta clase de ejercicio recibe el nombre de gramática o investigación gramatical. Este ejercicio evidenciará cómo funcionan las palabras y permitirá identificar la manera como deben o han de ser utilizados correctamente los conceptos en casos concretos, alejados de toda confusión.

En las Investigaciones Filosóficas Wittgenstein (1988), refiriéndose al carácter de su nueva propuesta de investigación para la filosofía, afirma en el $\int 90$ :

Nuestra investigación, sin embargo no se dirige a los fenómenos [...] Nos acordamos, quiere esto decir, del tipo de enunciados que hacemos sobre los fenómenos [...] Nuestro examen es por ello de índole gramatical. Y éste arroja luz sobre nuestro problema quitando de en medio malentendidos (p. 113).

La investigación gramatical no se encarga de analizar los fenómenos, sino que se dirige hacia los conceptos o enunciados que empleamos para referirnos a dichos fenómenos. No busca esen- cias ocultas, sino que revisa y describe la manera como dichos conceptos son empleados en casos particulares, los contextos que los envuelven y las reglas que de alguna manera se han establecido y que permiten hallar una regularidad en su uso, mostrando así su significado. De igual manera, en el estudio de los diferentes usos o contextos en los que el concepto es usado irá develando la forma correcta de usarlos y las situaciones en las que debido al desconocimiento y transgresión de estas reglas se cae en confusiones y malentendidos.

El método fijado por Wittgenstein para el trabajo filosófico consiste en aclaraciones acerca del funcionamiento de nuestras formas de expresión. Los problemas filosóficos no son problemas como los que trabaja la ciencia en la medida en que no son empíricos. Piénsese, por ejemplo, en conceptos como mente, alma, Dios, tiempo, entre otros. Como ya hemos venido esbozando, estos conceptos obtienen su significado en su uso regulado dentro de un juego de lenguaje concreto; cuando se violentan las reglas de los conceptos y se le dan usos inadecuados, connotaciones o tratamientos inapropiados, se cae inevitablemente en confusiones que dan origen a los supuestos problemas filosóficos.

Wittgenstein hace uso del concepto de gramática no en el sentido tradicional de armar correctamente una frase, sino en un sentido más amplio. No considera el lenguaje como un sistema de signos y reglas fijas y preestablecidas, sino como el uso de las palabras que está encarnado en la historia cotidiana de los individuos. El lenguaje no es algo separado o alejado de la vida de los hombres; hace parte de su vida diaria y se manifiesta en y desde ella. El lenguaje está ligado a la historia del hombre, a su sociedad, a su devenir histórico, empleando palabras del mismo Wittgenstein, a su "forma de vida". La gramática es la estructura de la práctica de nuestro uso del lenguaje $\mathrm{e}^{11}$.

11 "the concept of 'our practice of using language' is here intented to invoke the idea of language, not as 'some non-spatial, non-temporal phantasm', but as a 'spatial a temporal phenomenon' (Philosophy Investigation §108)" (McGinn, 1997, p. 14).

Polisemia No. 12, 102 - 112, La actividad filosófica según Wittgenstein. Confusiones conceptuales e investigación gramatical. Bogotá, ISSN: 1900-4648. Julio - Diciembre de 2011 
Con este ejercicio, Wittgenstein no pretende fijar reglas absolutas para el uso adecuado de los conceptos. Su propósito es evocar los diferentes modelos en los cuales son usados los conceptos y hacernos conscientes de esta gran variedad de usos; polifuncionalidad que constituye una característica inherente a los conceptos y al lenguaje mismo.

El método gramatical consiste entonces en recordar nosotros mismos los detalles de los distintos modelos de uso que constituyen lo que él llama "gramática de los conceptos". Su presentación no pretende instituirse como una teoría o metateoría. Su intención es mostrar los diferentes usos (significados) que adquieren los conceptos dentro de diferentes juegos de lenguaje ${ }^{12}$ $y$, de esta manera, prevenirnos de la pretensión de elevar de manera simplificadora un único uso general para los conceptos, independiente de contexto alguno y simultáneamente válido en cualquiera de ellos de manera absoluta. Con lo cual logra disolver las confusiones en el lenguaje emanada de la descontextualización y forcejeo de los conceptos para hacerlos encajar en otros contextos ajenos al propio. Cada juego de lenguaje posee su propia gramática o conjunto de reglas que determinan su propio funcionamiento.

Wittgenstein se opone así a una actitud teórica que intenta explicar, "penetrar el fenómeno". No hay nada qué explicar, la filosofía debe dejar las cosas como están pues la única obligación de la filosofía es describir el uso efectivo del lenguaje (Wittgenstein, 1988, p. 124). Esta actitud teórica, relacionada con el método y propósito científico, es el mayor obstáculo para la filosofía.

Los filósofos tienen constantemente ante los ojos el método de la ciencia y sienten una tentación irresistible a plantear y a contestar las preguntas del mismo modo que lo hace la ciencia. Esta tendencia es la verdadera fuente de la metafísica y lleva al filósofo a la oscuridad más completa (Wittgenstein, 1968, p. 46).

Cuando enfrentamos preguntas como qué es el significado, qué es el pensamiento, e intentamos darles respuesta de manera científica o teórica, nos vemos conducidos a una completa oscuridad. Wittgenstein no emite una crítica a la ciencia en general sino al uso del método o estilo científico para responder y/o preguntar acerca de cuestiones como éstas, ya que resulta inapropiado y erróneo el pretender buscar una explicación, descubrir y elaborar algo nuevo que revele su esencia ${ }^{13}$. Lo que se busca es la comprensión y claridad que ya está manifestada en nuestro uso de los conceptos y no en definiciones ni explicaciones precisas. La filosofía debe disolver sus problemas aclarando la manera como efectivamente son usados los conceptos en el lenguaje ordinario, sin tener que acudir a nuevas experiencia o a nueva información ${ }^{14}$.

\section{El problema de los filósofos}

Wittgenstein rechaza, según lo expuesto, la inclinación en la filosofía tradicional de hacer uso del método seguido en las ciencias. No está de acuerdo con esta visión cientificista que hasta el

12 Es relevante recordar que Wittgenstein afirma que el lenguaje funciona de manera similar a como lo hacen los juegos. Cada juego es diferente de otro en la medida en que cada uno contiene sus propias reglas que le dan su particularidad y su dinámica. Si un jugador no contemplase en sus acciones estas reglas, no estaría siguiendo ninguna clase de juego, o tal vez estaría realizando otra actividad diferente a la del juego que desea realizar. De igual manera, los conceptos se insertan en un juego de lenguaje con características propias y con reglas que lo determinan y lo definen. De estas reglas, los conceptos adquieren su significado; de la misma manera que se abandona un juego determinado cuando se cambian sus reglas, en el lenguaje, cuando se transgreden las reglas que lo conforman, se pasa a otro juego de lenguaje siendo probable el caer en confusiones ante el riesgo de sacar a los conceptos del contexto en el cual mantenía su significado, o simplemente, guiado por la tendencia a hacer funcionar un concepto en un contexto particular de la misma manera a como venía funcionando bajo otras circunstancias y con otras aplicaciones. Estos juegos de lenguaje son prácticas humanas que se insertan en una forma de vida.

13 "La filosofía expone meramente todo y no explica ni deduce nada. -Puesto que todo yace abiertamente, no hay nada que explicar. Pues lo que acaso esté oculto, no nos interesa" (Wittgenstein, 1988, pp. 126 - 127).

14 "Los problemas se resuelven no aduciendo nueva experiencia, sino compilando lo ya conocido. La filosofía es una lucha contra el embrujo de nuestro entendimiento por medio de nuestro lenguaje" (Wittgenstein, 1988, p. 123). "El trabajo del filósofo es compilar recuerdos para una finalidad determinada" (Wittgenstein, 1988, p. 131). 
momento han seguido algunos filósofos, quienes, en la pretensión de alcanzar el éxito que las ciencias y su método han logrado en el tratamiento de sus problemas, han querido extender dicho método a la filosofía sin tener en cuenta que ésta y la ciencia difieren en cuanto a sus objetos de estudio. La ciencia, por su parte, trabaja problemas empíricos, mientras que la filosofía trata cuestiones conceptuales. Es por esta razón que, en la filosofía, el método científico que se erige sobre la evidencia empírica fracasa cuando se intenta aplicar dentro de la labor filosófica.

Nuestro autor rechaza todo tipo de teoría explicativa, arguyendo que la filosofía debe dejar las cosas tal como están. La tarea del filósofo consiste en describir los fenómenos y 'ponerlos ante nuestros ojos'. Existe así una radical diferencia entre la tendencia a abordar los problemas filosóficos desde la explicación y el método descriptivo, el cual Wittgenstein considera que es el apropiado para la filosofía.

Ahora bien, el mayor obstáculo para realizar dicha investigación gramatical y aceptar la propuesta filosófica del segundo Wittgenstein consiste en abandonar ese viejo hábito epistemológico de crear teorías y explicaciones. Esta es una antigua forma de pensar que se encuentra enraizada en nuestro lenguaje, es, palabras más palabras menos, la forma de lenguaje que a diario empleamos y en la cual fuimos educados. Hace parte de nuestra vida.

Wittgenstein no duda en los Cuadernos Azul y Marrón en afirmar que aquello que hace más difícil el adoptar esta nueva línea de investigación filosófica es nuestra ansia de generalidad, que nos conduce a abordar los problemas e interpretarlos de una manera inadecuada (Wittgenstein, 1968, pp. 45-46). Esta tendencia surge y se manifiesta en dos aspectos fundamentalmente, los cuales se hallan muy relacionados. En primer lugar, existe una tendencia a homogeneizar los fenómenos, reflejada en el hábito de "buscar algo común a todas las entidades que usualmente incluimos bajo un término general" (Wittgenstein, 1968, p. 45). De esta forma, todos estos fenómenos son vistos como iguales, desconociendo sus particularidades. Tal homogeneización se basa en la creencia de la existencia de eso común y esencial en todos ellos, permitiendo incluirlos bajo un mismo conjunto con un mismo término.

Sin embargo, cuando nos enfrentamos con un problema filosófico en el que tenemos ante nuestros ojos un concepto que se desarrolla al interior de un juego de lenguaje y en el marco de unas circunstancias específicas y con propósitos determinados, es imposible dejar de lado estas particularidades para intentar asumir un conjunto de usos de un concepto como iguales. Los conceptos no pueden extraerse de su contexto pues su significado particular se aferra a este contexto y fuera de él es como si "les faltara el aire". Se pretende universalizar el concepto, extenderlo a todo contexto sin ser inscrito en ninguno en particular. El concepto es despojado de su capacidad de significar pues el sentido de un concepto surge del juego de lenguaje en el que se emplee y en donde convergen un sinnúmero de condiciones, formas de vida, particularidades que lo dotan de la riqueza de su significado.

Una segunda fuente del ansia de generalidad, y tal vez la principal, es la preocupación por el método de la ciencia basado en la explicación causal de los fenómenos de los que se ocupa (Wittgenstein, 1968, p. 46). Este sistema explicativo generaliza los fenómenos para poder construir sus grandes y complejas teorías, subsumiendo la particularidad de cada fenómeno bajo leyes generales. Las teorías explicativas validan esta generalización en la observación y corroboración de la correspondencia de los sistemas hipotéticos con los fenómenos reales. Por la misma naturaleza de la tradición filosófica, a ésta no le interesa el carácter hipotético pues ella busca la esencia y los primeros principios de las cosas para satisfacer su afán de elaborar teorías absolutas y definitivas que alberguen la verdad. De este modo, en la filosofía tradicional se halla una fuerte actitud teórica que conduce a cierto desprecio por lo particular.

Así, el ansia de generalidad que se manifiesta en la constante tendencia a elaborar explicaciones 
cada vez más exactas y universales, y que se encuentra presente en el método que se sigue en el momento de afrontar un problema de carácter filosófico, impide darles un adecuado tratamiento; suscitando de este modo los ya nombrados malentendidos o confusiones conceptuales que erróneamente reciben el nombre de problemas, pues como ya se ha enunciado, no son problemas empíricos, característica fundamental en los problemas científicos, sino que son confusiones gramaticales o conceptuales.

Que los problemas fuesen empíricos implicaría, entre otras peculiaridades propias, que como fenómenos pudiesen ser percibidos y observados, que tuviesen una conducta regular que pudiese ser analizada, registrada, sometida a pruebas y experimentos a partir de los cuales fuese posible plantear hipótesis e incluso permitiría postular y predecir estados y comportamientos que aún no han sido registrados. Todo esto es posible al interior de la ciencia gracias a la observación de la regularidad en la sucesión de estados de los fenómenos en circunstancias determinadas. Una vez planteada la hipótesis, se procederá a revisar su correspondencia o acierto con relación a lo que de hecho ocurre en el mundo real.

Esta situación de los fenómenos pertenecientes al ámbito científico constituye la principal diferencia con los problemas típicamente filosóficos, de carácter netamente conceptual. La clarificación de las reglas que rigen nuestro uso del lenguaje en distintos contextos no tiene relación alguna con lo empírico, con los hechos observables, que sí tiene la labor científica en particular en su tarea de dar explicaciones causales. Por tal razón, la filosofía deberá dejar de lado su tan acostumbrada ansia de generalidad en el intento por apropiarse del exitoso método científico y dedicarse a una tarea más acertada dentro de sus condiciones y posibilidades; ésta es la descripción de los usos de los conceptos en medio del funcionamiento del lenguaje.

\section{Referencias bibliográficas}

Arrington, R.L. \& H.-J. Glock (Eds.).(1991). Wittgenstein's Philosophical Investigation: Text and Context. Londres: Routledge.

Ayer, A.J. (1986). Wittgenstein. Barcelona: Crítica.

Bartley, W. W. (1987). Wittgenstein. Madrird: Cátedra.

Deursen van, C. A. (1973). Ludwing Wittgenstein. Introducción a su Filosofía. México: Carlos Lohle.

Fann, K.T. (1975). El Concepto de Filosofía en Wittgenstein. Madrid: Tecnos.
Holguin, M. (1992). La Filosofía como Critica del Lenguaje. En Ideas y Valores N87-88. . (1997). Wittgenstein y el Escepticismo. Universidad del Valle.

Hoyos, L. E. (Ed.). (2003). Lecciones de Filosofía. Bogotá: Universidad Externado de Colombia y Universidad Nacional de Colombia.

Kenny, A. (1994). Wittgenstein. Madrid: Alianza Editorial, Madrid.

$$
\text { (1990). El Legado de Wittgenstein. }
$$
México D.F: Siglo XXI. 
López, P. (1986). Introducción a Wittgenstein, Sujeto, Mente y Conducta. Barcelona: Editorial Herder.

McGinn, M. (1997). "Wittgenstein and Philosophical Investigations", Routledge Philosophy Guidebooks, University College London.

Meléndez, R. (1998). Verdad sin Fundamentos. Bogotá: Ministerio de Cultura.

Stern, D.G. (1995). Wittgenstein on Mind and Language. Nueva York: Oxford University Press.
Witgenstein L. (1968). Los Cuadernos Azul y Marrón. Traducción de Francisco Gracia Guillén. Madrid: Tecnos.

(1988). Investigaciones Filosóficas.

Traducción de Alfonso García Suárez y Ulises Moulines. México D.F: Critica.

(2003). Tractatus Logico-Philosophicus.

Traducción de Jacobo Muñoz e Isidoro Reguera. Madrid: Alianza. 